\title{
A Be star with a low nitrogen abundance in the SMC cluster NGC 330
}

\author{
D. J. Lennon ${ }^{1}$, J.-K. Lee ${ }^{2}$, P. L. Dufton ${ }^{2}$, and R. S. I. Ryans ${ }^{2}$ \\ 1 The Isaac Newton Group of Telescopes, Apartado de Correos 321, 38700 Santa Cruz de La Palma, Canary Islands, Spain \\ e-mail: dj1@ing.iac.es \\ 2 Department of Pure \& Applied Physics, The Queen's University of Belfast, BT7 1NN, Northern Ireland, UK
}

Received 13 July 2004 / Accepted 12 April 2005

\begin{abstract}
High-resolution UVES/VLT spectra of B 12, an extreme pole-on Be star in the SMC cluster NGC 330, have been analysed using non-LTE model atmospheres to obtain its chemical composition relative to the SMC standard star AV 304 . We find a general underabundance of metals which can be understood in terms of an extra contribution to the stellar continuum due to emission from a disk which we estimate to be at the $\sim 25 \%$ level. When this is corrected for, the nitrogen abundance for B 12 shows no evidence of enhancement by rotational mixing as has been found in other non-Be B-type stars in NGC 330, and is inconsistent with evolutionary models which include the effects of rotational mixing. A second Be star, NGC 330-B 17, is also shown to have no detectable nitrogen lines. Possible explanations for the lack of rotational mixing in these rapidly rotating stars are discussed, one promising solution being the possibility that magnetic fields might inhibit rotational mixing.
\end{abstract}

Key words. stars: abundances - stars: early-type - stars: emission-line, Be - galaxies: individual: Small Magellanic Cloud

\section{Introduction}

The open cluster NGC 330 has become one of the most studied young clusters in the Small Magellanic Cloud (SMC). There have been many spectroscopic studies of individual massive stars in the cluster, attention being concentrated on the most massive and luminous giants and supergiants (Lennon et al. 2003, 1996, 1993; Hill et al.1997; Hill 1999; Spite et al.1991). The most salient and interesting result from these studies is that all the B-type giants and supergiants are strongly nitrogen enriched. This striking result echoes the findings of Venn (1999), Dufton et al. (2000) and Trundle at al. (2004) that most earlytype supergiants in the SMC are enriched in nitrogen by around a factor of 10. It is also interesting to note that similarly large nitrogen enrichments have also been found for many, but not all, O-type stars in the SMC (Bouret et al. 2003; Hillier et al. 2003).

One of the most favoured explanations as to the cause of these enrichments is that rotationally induced mixing is responsible for polluting the atmospheres of these stars with $\mathrm{CN}$ processed material (Maeder \& Meynet 2001; Heger \& Langer 2000). In the context of stellar rotation, the cluster NGC 330 is again prominent since it has the highest known fraction of Be stars of any known cluster (Feast 1972; Grebel et al. 1996; Mazzali et al. 1996; Keller \& Bessell 1998). Indeed it has been suggested that this high incidence of Be stars might be linked to the low metallicity of the cluster (Maeder et al. 1999). This apparent dependence of the frequency of Be stars on metallicity has led to suggestions that stellar rotational rates might increase with decreasing metallicity, and hence increasing redshift, with far-reaching consequences for stellar evolution. For example, Meynet \& Maeder (2003) have suggested that stellar rotation might play a key role in the production of nitrogen in the Universe.

However, as discussed by Lennon (2003), Herrero \& Lennon (2004) and Walborn \& Lennon (2004), it is difficult to reconcile the predictions of various theories with the observed distributions of stellar rotational velocities, or the observed distribution of massive stars in the HR diagram. Lennon (2003) discussed the case of NGC 330 and it seems clear that, even allowing for inclination effects, the rotational velocities of the nitrogen enriched B-type giants are much lower than those needed to produce enrichment in the models. A major concern for any inferences drawn about the role of rotation on massive star evolution is that there is a very strong observational bias towards low- $v \sin i$ stars, for the simple reason that spectra with even moderate rotational velocities are extremely difficult to analyse. Furthermore, from studies of the distribution of rotational velocities of massive stars (Wolff \& Simon 1997; Day \& Warner 1975) it seems reasonable to assume that the majority of the low- $v \sin i$ stars are indeed slow rotators. Ideally one needs to consider pole-on stars which are known to have high rotational velocities. However deducing rotational velocities from the spectra of narrow-lined normal B-type stars is difficult, whereas pole-on Be stars fit this description extremely well. They are acknowledged to be fast rotators and the 
orientation angle of the rotation axis may be deduced from the morphology of the disk emission lines, rather than just the widths of absorption lines.

Although the probability of observing an extreme pole-on star is low, the very high fraction of Be stars in the cluster NGC 330 makes this more likely. Furthermore Baade et al. (2002) have coincidentally carried out a search for spectral variability with UVES/VLT in two low- $v \sin i$ Be stars in the cluster, one of which, B 12 (Robertson 1970), is almost poleon with a $v \sin i$ of only $40 \mathrm{~km} \mathrm{~s}^{-1}$. Lennon et al. (1993) have also noted that this Be star had a low $v \sin i$, as had Hummel et al. (2001) in a FORS/VLT study of Be stars in the cluster. In this paper we present a description of the high resolution spectrum of B 12 obtained by Baade et al., and attempt a detailed analysis of the abundances using non-LTE model atmospheres, paying special attention to the nitrogen lines in its spectrum.

\section{Observational data}

The data for B12, which were kindly made available to us by Thomas Rivinius, are described in detail by Baade et al. (2002), and consist of 23 individual observations of B 12 using UV-Visual Echelle Spectrograph (UVES) on the VLT. All 23 spectra, each of which had a $S / N$ ratio of $\approx 80$, were coadded as Baade et al. found little evidence for variability. It was immediately clear that the spectrum of B 12 is peculiar compared to those of the other B-type stars in NGC 330 observed by Lennon et al. (2003) in that the N II spectrum was extremely weak. This is illustrated in Fig. 1 where we compare the spectral region containing the $\mathrm{N}_{\mathrm{II}}$ lines at 3995, 4041 and $4043 \AA$ with that of other SMC B-type stars; NGC 330-B 32 (also observed by Baade et al), NGC 330-B 04 (observed with UVES/VLT by DJL), and the standard AV 304 (observed with UVES/VLT by Rolleston et al. 2003). It is clear from this figure that the $\mathrm{N}$ II lines are at the limit of detectability in the spectrum of B 12 despite its very high $S / N$ ratio. The other B-type stars in NGC 330 are in sharp contrast to B 12, but note that Lennon et al. (2003) found these objects to be nitrogen rich with respect to the standard AV 304 by approximately a factor of 10 . This is a surprising result as it implies that nitrogen abundance of B 12 must be very low, as can be seen by a comparison with the spectrum of AV 304 (note that the N II line at $3995 \AA$ is only visible in this star because of its very low projected rotational velocity).

While absorption lines of N II are mostly absent from the spectrum of B 12, many other metal absorption lines typical of an early B-type star are present; in particular there are absorption lines due to O II, Si III, Mg II and C II. We also note the presence of several emission lines due to allowed transitions of Fe II and Si II. The equivalent widths $(E W s)$ of the metal absorption lines were measured using a STARLINK spectral analysis program DIPSO (Howarth et al. 1996). Non-linear least square fitting routines were used with Gaussian profiles being adopted for the absorption lines and low order polynomials for the adjacent continuum regions. This procedure also yielded an estimate of the radial velocity of $152 \pm 5 \mathrm{~km} \mathrm{~s}^{-1}$. For the hydrogen lines, the profiles were fitted with the continuum levels being defined at $\pm 16 \AA$ from the line centre. The

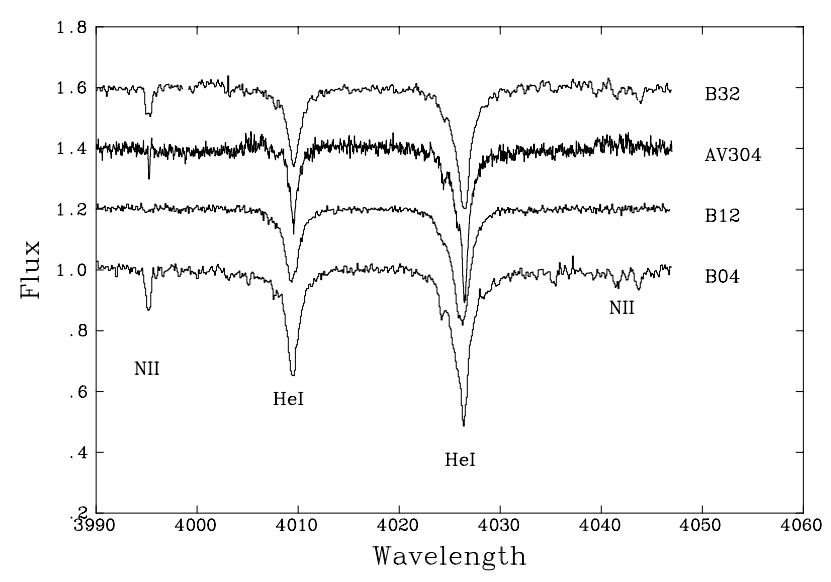

Fig. 1. A comparison of the 3990-4050 A regions for the nitrogen rich stars B 32 and B 04 in NGC 330, the nitrogen normal star AV304, and the Be star B 12. We indicate the presence of the He I lines at 4009 and $4026 \AA$, together with the N II lines at 3995, 4041 and $4043 \AA$. Note that the extraordinary sharpness of the lines in AV304 permits the secure identification on the $\mathrm{N}$ II line at $3995 \AA$ while the high $S / N$ ratio of the data for B12 permits a tentative detection of this same line, which is significantly weaker than in the other NGC 330 stars B 04 and B 32 .

Table 1. Equivalent widths (mÅ) for B 12. Typical measurement uncertainties is $<5 \mathrm{~mA}$.

\begin{tabular}{lrlrlr}
\hline \hline Line & \multicolumn{1}{l}{$E W \mathrm{~s}$} & Line & EWs & Line & $E W \mathrm{~s}$ \\
\hline C II & & O II & & Si II & \\
3918.98 & 55 & 4132.81 & 13 & 4128.07 & $\leq 10$ \\
3920.68 & 56 & 4253.90 & 28 & 4130.89 & $\leq 10$ \\
4074.52 & $\leq 10$ & 4317.14 & 22 & & \\
4267.02 & 91 & 4319.63 & 22 & Si III & \\
& & 4349.43 & 22 & 4552.38 & 59 \\
N II & & 4395.94 & 11 & 4567.82 & 50 \\
3995.00 & 12 & 4414.90 & 35 & 4574.86 & 38 \\
4601.48 & $\leq 10$ & 4416.97 & $7:$ & & \\
4607.16 & $\leq 10$ & 4452.37 & $9:$ & Si IV & \\
4621.29 & $\leq 10$ & 4590.97 & 26 & 4088.85 & 10 \\
4643.09 & $\leq 10$ & 4595.96 & 16 & 4116.10 & $\leq 10$ \\
& & 4609.44 & $6:$ & & \\
O II & & 4613.11 & $\leq 10$ & Fe II & \\
3954.37 & 16 & 4638.86 & 21 & 4395.78 & 12 \\
4069.62 & 35 & 4641.82 & 35 & & \\
4072.16 & 25 & 4649.14 & 37 & Mg II & \\
4075.85 & 28 & 4650.84 & 13 & 4481.13 & 43 \\
4078.84 & $\leq 10$ & 4661.63 & 13 & & \\
4083.91 & $\leq 10$ & 4676.24 & 13 & Al II & \\
4084.65 & 9 & 4699.00 & $9:$ & 4528.91 & $13:$ \\
4087.16 & $\leq 10$ & 4705.35 & 11 & & \\
4089.29 & 10 & & & & \\
\hline
\end{tabular}

equivalent widths are listed in Table 1 . Generally the lines with $E W \mathrm{~s} \geq 15 \mathrm{~m} \AA$ were well observed, with convincing fits being obtained for several weaker lines. 
Table 2. Atmospheric parameters and abundances of AV 304 together with the differential abundances derived for B 12 with the number of lines considered being given in parentheses. The errors for the former are taken from Hunter et al. (2005). For the latter they are the standard error among the lines of the same element; no error is given for $\mathrm{N} \mathrm{II}$ and $\mathrm{Mg}$ II since only one feature contributed to these abundances. Also listed in this table are the parameters and abundances which one would obtain for B 12 assuming a disk was contributing $25 \%$ to the continuum flux. There are two sets of stellar parameters for each disk/no disk model; in each case the higher effective temperature is the one derived by taking the mean of the upper and lower limits set by the absence of the Si IV and Si II lines, while the lower value represents the effective temperature set by the absence of the Si II lines only.

\begin{tabular}{|c|c|c|c|c|c|c|c|}
\hline & \multirow{2}{*}{\multicolumn{2}{|c|}{ AV $304^{*}$}} & \multicolumn{5}{|c|}{ B 12} \\
\hline & & & No disk & No disk & $25 \%$ disk & $25 \%$ disk & \\
\hline$T_{\text {eff }}(\mathrm{K})$ & 27500 & & 23000 & 21500 & 24000 & 22500 & \\
\hline $\log g\left(\mathrm{~cm} \mathrm{~s}^{-2}\right)$ & 3.90 & & 3.60 & 3.45 & 4.00 & 3.85 & \\
\hline$v_{\mathrm{t}}\left(\mathrm{km} \mathrm{s}^{-1}\right)$ & 3 & & 3 & 3 & 3 & 3 & \\
\hline $\mathrm{C}_{\text {II }}$ & $7.36 \pm 0.12$ & (3) & $-0.06 \pm 0.02$ & $-0.15 \pm 0.04$ & $0.19 \pm 0.02$ & $0.09 \pm 0.04$ & (2) \\
\hline N II & 6.55 & (1) & -0.35 & -0.33 & -0.19 & -0.17 & (1) \\
\hline O II & $8.13 \pm 0.10$ & (42) & $-0.62 \pm 0.13$ & $-0.43 \pm 0.14$ & $-0.35 \pm 0.12$ & $-0.19 \pm 0.10$ & (13) \\
\hline Mg II & 6.77 & (1) & -0.33 & -0.44 & -0.14 & -0.24 & (1) \\
\hline Si III & $6.76 \pm 0.18$ & (4) & $-0.43 \pm 0.12$ & $-0.27 \pm 0.12$ & $-0.05 \pm 0.10$ & $0.08 \pm 0.09$ & (3) \\
\hline
\end{tabular}

${ }^{*}$ Hunter et al. (2005). Rolleston et al. (2003) derived $T_{\text {eff }}=27500 \mathrm{~K}, \log g=3.8, v_{\mathrm{t}}=5 \mathrm{~km} \mathrm{~s}^{-1}$ in their LTE analysis.

\section{Method of analysis}

We have already referred to the marked weakness of the nitrogen lines in B 12 compared to other nitrogen rich B-type giants in NGC 330. Since it is generally accepted that Be stars are B-type stars near the end of the main-sequence it is especially interesting to try to quantify the atmospheric nitrogen abundance of B 12 in order to see to what degree, if any, the nitrogen content has been enhanced by rotational mixing. In the following subsections we present the results of a non-LTE model atmosphere analysis of B 12 relative to a B-type SMC standard. Clearly the use of plane-parallel models for a star like $\mathrm{B} 12$ which is a fast rotator with a disk is questionable. However we consider it a reasonable first approximation and return to the question of its reliability in the subsequent discussion.

\subsection{Model atmosphere calculations}

The analysis is based on grids of non-LTE model atmospheres calculated using the codes TLUSTY and SYNSPEC (Hubeny 1988; Hubeny \& Lanz 1995; Hubeny et al. 1998). Details of the methods can be found in Ryans et al. (2003), while the grids are discussed in more detail by Dufton et al. (2005) and Lee et al. (2004).

Briefly we used a grid appropriate to the SMC metallicity that has been generated with an iron abundance of $\left[\frac{\mathrm{Fe}}{\mathrm{H}}\right]=6.8$, i.e. 0.7 dex less than Galactic. The model grid covers a range of effective temperatures from 12000 to $35000 \mathrm{~K}$, logarithmic gravities from 4.5 dex down to close to the Eddington limit and microturbulences from 0 to $30 \mathrm{~km} \mathrm{~s}^{-1}$. For any set of atmospheric parameters, five models were then calculated keeping the iron abundance fixed but allowing the light element (e.g. $\mathrm{C}, \mathrm{N}, \mathrm{O}, \mathrm{Mg}$ and $\mathrm{Si}$ ) abundances to vary from +0.8 dex to -0.8 dex around their base values. These models are then used to calculate spectra, which provide theoretical hydrogen and helium line profiles and equivalent widths for light metals for a range of abundances. The theoretical equivalent widths were measured using IDL programs to integrate the flux within a certain wavelength range chosen to include the line of interest.

\subsection{Comparison star}

Hunter et al. (2005) have analysed UVES/VLT spectroscopy (Rolleston et al. 2003) of an SMC main sequence star, AV 304. They used the same grid of TLUSTY model atmospheres as discussed above, together with similar techniques to those adopted here to estimate the atmospheric parameters and element abundances (see Sects. 3.3 and 3.4). As such, these results provide a useful comparison to those for B12 and we summarize the results of their analysis in Table 2.

\subsection{Estimation of atmospheric parameters}

The atmospheric parameters, surface gravity and effective temperature, are inter-related, and their derivation requires an iterative approach. We adopted an appropriate value of the surface gravity and then estimated the effective temperature using the silicon ionization balance. This temperature estimate was then used to deduce a new value for the surface gravity from fitting the Balmer lines, and the process iterated to convergence. Unfortunately in the spectra of B 12, it was not possible to distinquish either Si II or Si IV lines. However their absence effectively provided constraints on the lower and upper limits of the effective temperature. The mean of the derived lower and upper limits, which differ by approximately $2500 \mathrm{~K}$, was then taken to be the stellar effective temperature, although we will discuss below the effect of using these upper and lower bounds on the derived abundances. There were insufficient metal lines to determine the microturbulent velocity and we have therefore adopted the value of Hunter et al. (2005) of $v_{\mathrm{t}}=3 \mathrm{~km} \mathrm{~s}^{-1}$. We note that due to the weakness of the metal lines in the spectrum 
of B 12, the abundance estimates are insensitive to the value adopted for the microturbulence.

\subsection{Abundance estimates}

The metal lines were used, along with the atmospheric parameters derived above, to deduce absolute abundances for B 12. A typical observational uncertainty of $\leq 5 \mathrm{~m} \AA$ in the $E W$ estimate of an individual line would result in an abundance error less than 0.1 dex. Normally only metal lines with an $E W$ of greater than $15 \mathrm{~m} \AA$ were considered. However for the ion N II, the only feature observed was at $3995 \AA$ and had an equivalent width of $12 \mathrm{~m} \AA$. We have therefore included this line but note that the corresponding nitrogen abundance should be considered as an upper limit. A detailed line-by-line differential analysis (summarised in Table 2) of B 12 was then carried out relative to AV 304, as this should minimise the effect of systematic errors.

\section{Discussion}

The most important and obvious result of this investigation is that B 12 does not exhibit any evidence for the kind of nitrogen enrichments found in other evolved B-type stars in NGC 330. Surprisingly however all elements in B 12 show a general under-abundance relative to AV 304 and other NGC 330 stars (Lennon et al. 2003). For example compared with AV 304, the under-abundances range from $-0.06 \mathrm{dex}$ for $\mathrm{C}$ to $-0.62 \mathrm{dex}$ for $\mathrm{O}$, with $\mathrm{N}, \mathrm{Mg}$ and $\mathrm{Si}$ showing rather similar underabundances of -0.35 to -0.45 dex.

Of course our estimate of the effective temperature is derived by simply taking the mean of the upper and lower bounds implied by the absence of Si IV and Si II lines. While the effective temperature could lie anywhere within this range, increasing the effective temperature (and, for consistency, the surface gravity) only increases the discrepancy between B 12 and AV 304. Decreasing our effective temperature to its lower limit of $21500 \mathrm{~K}$ does improve the overall situation for all elements (see Table 2) but still leaves us with significant systemic differences with AV 304. There are two likely causes of these differences which are suggested by the Be nature of B 12; veiling of the continuum due to a disk, and departures from standard non-rotating plane parallel models caused by rapid rotation.

\subsection{Disk contribution?}

Our program star B 12 is an extreme Be star which has very strong $\mathrm{H} \alpha$ emission from a face-on disk. One can easily compute the degree of continuum veiling for a given absorption line, which is required to give a normal SMC abundance. If the fractional disk contribution to the continuum is defined as $x$, then the relationship between the measured $E W \mathrm{~s}, w$, and its intrinsic $E W \mathrm{~s}$ without a disk, $w_{0}$, will be given as $(1+x)=\left(w_{0} / w\right)$. In practice, $w_{0}$ can be obtained by finding an $E W$ that gives the same abundance as AV 304. Such scaling factors for each line are shown in Fig. 2. The error-bars are calculated by assuming the measurement error of B 12 is similar to that in AV304 and thus only reflects the uncertainty in the observational measurement. No noticable dependence of $x$ by wavelength is seen in

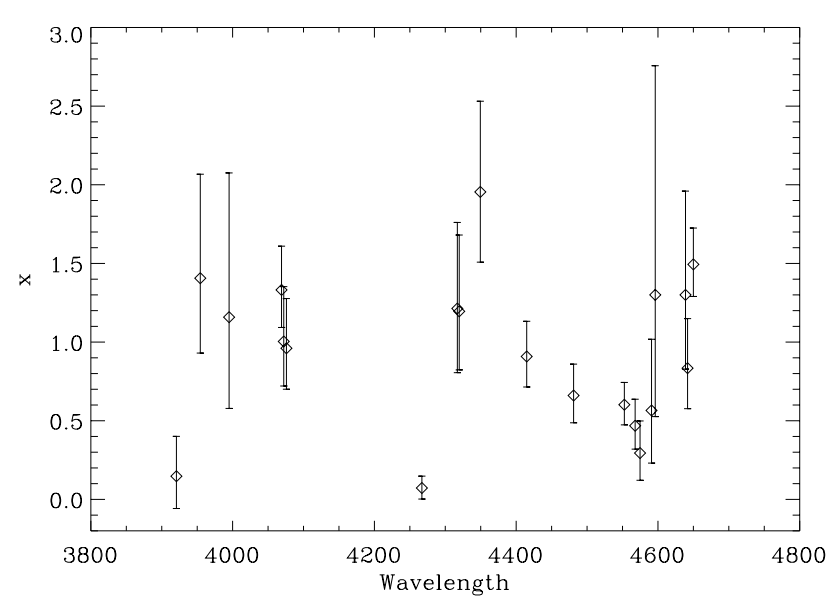

Fig. 2. The fractional contribution, $x$, of the disk relative to the stellar continuum which is required for metal lines in B 12 to have the same abundance as in the comparison star AV 304. These calculations have been performed for the $T_{\text {eff }}=23000, \log g=3.60$ model from Table 2 .

Fig. 2, and the mean value of $x$ is $0.94 \pm 0.49$, implying half of the total flux comes from the disk. The cooler model for B 12 gives a lower mean value for $x$ of $0.60 \pm 0.32$, implying that $40 \%$ of the total flux may be contributed by the disk.

Could a disk contribute so much flux? There is observational evidence that the contribution of the disk can be quite significant. For example, Telting et al. (1998) have carried out a long-term photometric monitoring of X Persei, a Be star whose disk is continuously being destroyed and reformed. This provides a good opportunity to observe the star with and without the disk and thus to estimate the disk contribution. They showed that the $V$ magnitude can change by 0.6 to 0.7 mag, a little less in B, which implies that the disk may contribute up to $40 \%$ of the total flux. This supports our assumption and leaves open the possibility that the abundances of metal lines in B 12 are in fact "normal" but appear to be under-abundant due to continuum contamination.

In a further refinement of this model we have undertaken a full model atmosphere analysis for varying fractions of disk contribution, taking into account the change in effective temperature and surface gravity implied by the disk-subtracted and re-normalized spectrum. It is important to note that the derived surface gravity increases as the amount of continuum subtraction increases, as does the effective temperature. The increased gravity leads to a decrease in the theoretical metal line strengths and an even stronger increase in the abundance estimates (summarised in Table 2). Furthermore, the Balmer lines enable us to estimate the maximum continuum contribution by using the goodness of fit to their line cores, as shown in Fig. 3. Compared to the simple approximation of simply scaling line strengths, discussed above, this procedure reduces the fraction of veiling necessary to obtain "normal" abundances, although as Fig. 3 shows, increasing the veiling beyond $25 \%$ leads to unacceptable fits to the Balmer lines. In Table 2 we list the abundances which result from assuming a disk contribution of $25 \%$ for both estimates of the effective temperature discussed above. We can see that this approach for B 12 results in only moderate 

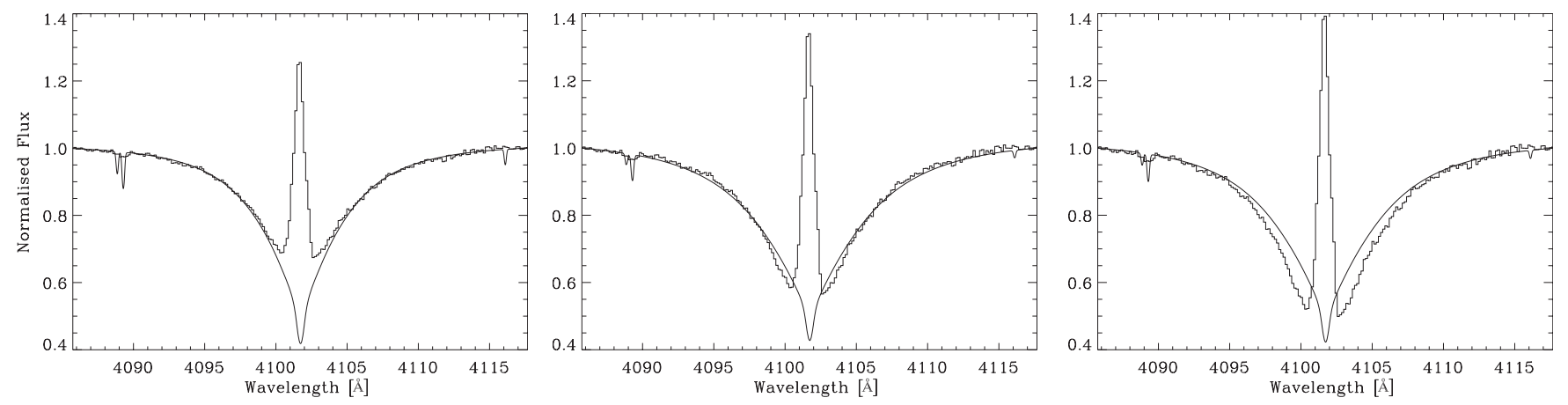

Fig. 3. $\mathrm{H} \delta$ lines with the zero, $25 \%$ and $35 \%$ continuum disk contribution and the fits by theoretical profiles modelled with the atmospheric parameters corresponding to the low $T_{\text {eff }}$ case as presented in Table 2 . Parameters for the $35 \%$ model are $T_{\text {eff }}=22750 \mathrm{~K}$ and $\log g=3.95$.

discrepancies with AV 304, particularly for the lower effective temperature model.

\subsection{Rapid rotation?}

Be stars are generally assumed to be fast-rotators, so it is important to investigate whether or not our analysis, which is based upon non-rotating models, is valid. The answer to this question hinges on the issue of the true rotational velocity of B 12. As has been shown by a number of authors (Collins et al. 1991; Howarth \& Smith 2001), the effect of rotation (through von Zeipel gravity darkening) on line profiles of OB stars only becomes sigificant for rotational velocities greater than about $90 \%$ of the critical value. Recently Chauville et al. (2001) analysed a sample of $116 \mathrm{Be}$ stars and concluded that most Be stars rotate at $80 \%$ of their critical velocity, with a very low dispersion around this value. Such a rotational velocity is too low to have a serious impact on line strengths. However this result has been questioned by Townsend et al. (2004) who show that stars rotating very close to their critical velocities will have their rotational velocities underestimated using the standard line-width techniques used by Chauville et al. Essentially the line widths of B-type stars are insensitive to rotational velocity for values above about $80 \%$ of the critical velocity. As Townsend et al. (2004) point out, this raises the possibility that B-type stars rotate much closer to their critical velocities than is generally believed. If this is true for B 12 then it may well be that the residual differences in abundances between B12 and AV304 (shown in Table 2), and the discrepancies in fitting the Balmer line cores illustrated in Fig. 3, result from the neglect of these effects.

\subsection{Evolutionary status}

It is very difficult to estimate the luminosity and hence the mass of B12 due to both the contribution of the disk to the observed magnitudes, and the unknown rotational velocity of the star (a rapidly rotating pole-on star will appear brighter). Nevertheless a typical mass for a main-sequence Bstar with the temperature and surface gravity of B 12 is $\sim 10$ $M_{\odot}$, so it is not unreasonable to assume that the mass of B 12 lies near to this value. Maeder \& Meynet (2001) have published an evolutionary track for a $9 M_{\odot}$ star with SMC-like composition and an initial rotational velocity of $300 \mathrm{~km} \mathrm{~s}^{-1}$, well below the critical value which is above $450 \mathrm{~km} \mathrm{~s}^{-1}$. As discussed by Lennon (2003), this model implies a surface nitrogen abundance of approximately 7.2 dex by the time it reaches the end of the main-sequence, and an equatorial velocity of approximately $200 \mathrm{~km} \mathrm{~s}^{-1}$. Such a large nitrogen enhancement is clearly ruled out by the current observations. Furthermore, since B 12 may be rotating even faster than this model implies, the abundance discrepancy is probably being underestimated by this comparison.

Why does B 12 have no nitrogen enrichment? It may well be the case that rotational mixing is less efficient than current models predict, in which case B 12 can be used to provide a strong constraint on the models. This seems unlikely given the results of Lennon et al. (2003) for other B-type stars in the cluster, plus the other results for early-type stars mentioned in the introduction. Is it possible that B 12 was a slow rotator which has "recently" been spun-up? Given the large number of Be stars in NGC 330, a spin-up mechanism such as mass-transfer in a binary system, needs to be quite common and co-ordinated in time, which seems unlikely.

Perhaps a more attractive solution lies in the recent work on the influence of magnetic fields with rotation on stellar evolution. The idea that OB stars have magnetic fields has steadily gained credence in recent years with the direct detection of magnetic fields in a handful of stars such as $\beta$ Cephei (B1 III, $B \sim 360 \mathrm{G}$, Donati et al. 2001) and $\theta^{1}$ Orionis $\mathrm{C}(\mathrm{O} 7 \mathrm{Vp}$, $B \sim 1100 \mathrm{G}$, Donati et al 2002), supplementing abundant indirect evidence provided for example by X-rays (see Schulz et al. 2003, for a recent discussion of Chandra data for the Orion Trapezium stars). Neiner et al (2003) have also reported on the detection of a magnetic field $(B \sim 530 \mathrm{G})$ in the Be star $\omega$ Orionis (B2 IIIe). An encouraging model for explaining the existence of magnetic fields is that they are created as a result of a dymano action in the convective core (Charbonneau \& MacGregor 2001; MacGregor \& Cassinelli 2003) or envelope (MacDonald \& Mullan 2004; Mullan \& MacDonald 2005), models which have reasonable success in predicting the observed range of surface field strengths. Maeder \& Meynet (2004) have discussed the evolution of the suface abundances in the presence of magnetic fields and rotation and although their results are preliminary, their $15 M_{\odot}$ model implies that magnetic fields may inhibit mixing of core-processed material 


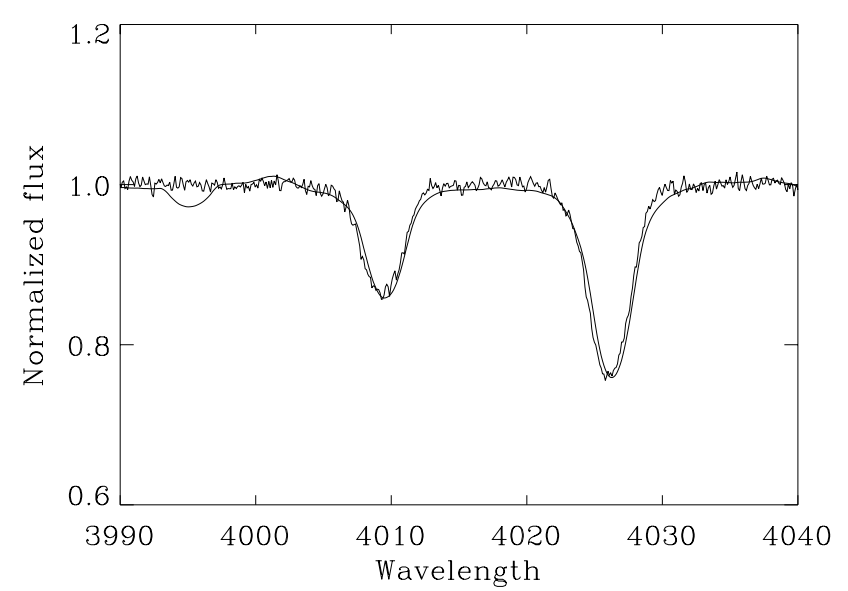

Fig. 4. Comparison of N II $3995 \AA$ region of the spectra of the Be star B 17 with the nitrogen rich B-type star B 32 (smooth line), the latter having been convolved with a rotational broadening fuction of $140 \mathrm{~km} \mathrm{~s}^{-1}$. Note that the nitrogen line in question is clearly absent in B 17.

to the surface and further that such models may rotate as solid bodies while on the main-sequence. The latter point is important since compared to rotating models without magnetic fields, the models with magnetic fields are rotating faster at the end of the main sequence.

Both of these characteristics, fast rotation and a lack of evidence of mixing, are relevant to B 12 . However, is the lack of mixing typical of Be stars? This is a difficult question to answer since most Be stars have such large projected rotational velocities that the upper limits on detection of the N II spectrum at typical values of $\mathrm{s} / \mathrm{n}$ provide no meaningful constraints. Although a magnetic field was detected in the Be star discussed by Neiner et al (2003), and these authors claim it to be nitrogen rich, examination of their abundances show that the star is mildly metal rich and in fact its carbon-to-nitrogen ratio is normal for nearby galactic B-stars (see Herrero \& Lennon 2004). We also note that Baade et al. (2002) also obtained 25 UVES/VLT observations of B 17, another Be star NGC 330 which has only a moderately high $v \sin i$ of $140 \mathrm{~km} \mathrm{~s}^{-1}$. We have therefore compared its spectrum with that of the nitrogen rich B-star B 32, which appears to be a good spectral analogue, although we have convolved the spectrum of B 32 with a rotational broadening function of $v \sin i=140 \mathrm{~km} \mathrm{~s}^{-1}$. The nitrogen lines are clearly absent in B 17 (Fig. 4). It is therefore tempting to suggest that a characteristic of Be stars is that they have magnetic fields which constrains them to rotate as solid bodies and inhibits mixing. Of course the existence of magnetic fields in Be stars is a very attractive scenario from the point of view of explaining the existence of the disk itself through magnetic compression (Porter \& Rivinius 2003), although some doubt has been cast on this mechanism by Owocki $\&$ ud Doula (2003).

\section{Conclusion}

We have investigated the spectrum of an extreme pole-on $\mathrm{Be}$ star NGC 330-B 12 in the SMC and found it to be almost devoid of nitrogen lines. In fact its spectrum is consistent with that of the normal SMC star AV 304 and it is inconsistent with the predictions of stellar evolution models which include the effects of rotationally induced mixing. We speculate that our findings lend support to the idea that magnetic fields may be present in Be stars, inhibiting mixing, and that the star is also rotating at a velocity close to its critical value which in turn leads to some residual moderate abundance anomalies, an artifact of models not including the effect of von Zeipel gravity darkening. Clearly, the SMC's very low pristine nitrogen abundance make it the ideal place in which to search for nitrogen enhancements in fast rotators, and in Be stars.

Acknowledgements. J.K.L. acknowledges financial support from PPARC (grant No. G/O/2001/00173), and D.J.L. acknowledges support through QUB's visiting fellow programme (grant No. V/O/2000/00479). We are indebted to Thomas Rivinius and Dietrich Baade for providing us with their UVES spectra of Be stars in NGC 330, and Ian Hunter for making available his analysis of AV 304.

\section{References}

Baade, D., Rivinius, Th., Stefl, S., \& Kaufer, A. 2002, A\&A, 383, 31 Bouret, J.-C., Lanz, T., Hillier, D. J., et al. 2003, ApJ, 595, 1182 Charbonneau, P., \& MacGregor, B. 2001, ApJ, 559, 1094

Chauville, J., Zorec, J., Ballereau, D., et al. 2001, A\&A, 378, 861

Collins, G. W., Truax, R. J., \& Cranmer, S. R. 1991, ApJS, 77, 541

Day, R. W., \& Warner, B. 1975, MNRAS, 173, 419

Donati, J.-F., Wade, G. A., Babel, J., et al. 2001, MNRAS, 326, 1265

Donati, J.-F., Babel, J., Harries, T. J., et al. 2002, MNRAS, 333, 55

Dufton P. L., McErlean, N. D., Lennon, D. J., \& Ryans, R. S. I. 2000, A\&A, 353, 311

Dufton, P. L., Ryans, R. S. I., Trundle, C., et al. 2005, A\&A, 434, 1125

Feast., M. W. 1972, MNRAS, 159, 113

Grebel, E. K., Roberts, W. J., \& Brandner, W. 1996, A\&A, 311, 470 2003, MNRAS, 339, 157

Heger, A., \& Langer, N. 2000, ApJ, 544, 1016

Herrero, A., \& Lennon, D. J. 2004, Stellar Rotation, ed. A., Maeder, \& P. Eenens, (ASP), IAU Symp., 215, 209

Hill, V., Barbuy, B., \& Spite, M. 1997, A\&A, 323, 461

Hill, V. 1999, A\&A, 345, 430

Hillier, D. J., Lanz, T., Heap, S. R., et al. 2003, ApJ, 588, 1039

Howarth, I. D., Murray, J., Mills, D., et al. 1996, STARLINK User Note SUN 50, Rutherford Appleton Laboratory/CCLRC

Howarth, I. D., \& Smith, K. C. 2001, MNRAS, 327, 353

Hubeny, I. 1988, Computer Phys. Comm., 52, 103

Hubeny, I., \& Lanz, T. 1995, ApJ, 439, 875

Hubeny, I., Heap, S. R., \& Lanz, T. 1998, in Boulder-Munich: Properties of Hot, Luminous Stars, ed. I. D. Howarth, ASP Conf. Ser., 131, 108

Hummel, W., Gässler, W., Muschielok, B., et al. 2001, A\&A, 371, 932 Hunter, I., Dufton, P. L., Ryans R. S. I., et al. 2005, A\&A, 436, 687

Keller, S. C., \& Bessell, M. S. 1998, A\&A, 340, 397

Lee, J.-K., Dufton P. L., Ryans, R. S. I., \& Rolleston W. J. R. 2004, A\&A, submitted

Lennon, D. J. 2003, A Massive Star Odyssey, ed. K. A., van der Hucht, A., Herrero, \& C., Esteban (ASP), IAU Symp., 212, 308

Lennon, D. J., Dufton, P. L., Mazzali, P. A., et al. 1993, Space Sci. Rev., 66, 169

Lennon, D. J., Dufton, P. L., Mazzali, P. A., et al. 1996, A\&A, 314, 243

Lennon, D. J., Dufton, P. L., \& Crowley, C. 2003, A\&A, 398, 455

MacDonald, J., \& Mullan, D. J. 2004, MNRAS, 348, 702 
MacGregor, K. B., \& Cassinelli, J. P. 2003, ApJ, 586, 480

Maeder, A., Grebel, E., \& Mermilliod, J. C. 1999, A\&A, 346, 459

Maeder, A., \& Meynet, G. 2001, A\&A, 373, 555

Maeder, A., \& Meynet, G. 2004, A\&A, 422, 225

Mazzali, P. A., Lennon, D. J., Pasian, F., et al. 1996, A\&A, 316, 173

Meynet, G., \& Maeder, A. 2003, A\&A, 404, 975

Mullan, D. J., \& MacDonald, J. 2005, MNRAS, 356, 1139

Neiner, C., Hubert, A.-M., Fremat, Y., et al. 2003, A\&A, 409, 275

Owocki, S. P., \& ud Doula, A. 2003, in Magnetic fields in O, B and A stars, ed. L. A., Balona, H. F., Henrichs, R., Medupe, ASP Conf. Ser., 305, in press [arXiv: astro-ph/0310179]

Porter, J. M., \& Rivinius, T. 2003, PASP, 115, 1153

Robertson, J. W. 1974, A\&AS, 15, 261

Rolleston, W. J. R., Venn, K. A., Tolstoy, E., \& Dufton, P. L. 2003, A\&A, 400, 21
Ryans, R. S. I., Dufton P. L., Mooney, C. J., et al. 2003, A\&A, 401, 1119

Schulz, N. S., Canizares, C., Huenemoerder, D., \& Tibbets, K. 2003, ApJ, 595, 365

Spite, F., Richtler, T., \& Spite, M. 1991, A\&A, 252, 557

Telting, J. H., Waters, L. B. F. M., Roche, P., et al. 1998, MNRAS, 296, 785

Townsend, R. H. D., Owocki, S. P., \& Howarth, I. D., 2004, MNRAS, 350,189

Trundle, C., Lennon, D. J., Puls, J., \& Dufton, P. L. 2004, A\&A, 417, 217

Venn, K. A. 1999, ApJ, 518, 405

Walborn, N. R., \& Lennon, D. J. 2004, Stellar Rotation, ed. A., Maeder, \& P., Eenens (ASP), IAU Symp., 215, 512

Wolff, S., \& Simon, T. 1997, PASP, 109, 759 